\title{
Earthquake Barcode from a Single-Degree-of-Freedom System
}

\author{
Chein-Shan Liu1, Chih-Wen Chang2 \\ ${ }^{1}$ Department of Civil Engineering, National Taiwan University, Taipei, Taiwan \\ ${ }^{2}$ Cloud Computing and System Integration Division, National Center for High-Performance Computing, \\ Taichung, Taiwan \\ Email: liucs@ntu.edu.tw
}

Received 4 November 2014; revised 12 December 2014; accepted 29 December 2014

Copyright (C) 2015 by authors and Scientific Research Publishing Inc.

This work is licensed under the Creative Commons Attribution International License (CC BY).

http://creativecommons.org/licenses/by/4.0/

(c) (i) Open Access

\section{Abstract}

Earthquake is a violent and irregular ground motion that can severely damage structures. In this paper we subject a single-degree-of-freedom system, consisting of spring and damper, to an earthquake excitation, and meanwhile investigate the response behavior from a novel theory about the dynamical system $\dot{x}=f(x, t)$, by viewing the time-varying signum function of $\|f\|^{2}\|x\|^{2}-2(f \cdot x)^{2}$. It can reflect the characteristic property of each earthquake through $(x(t), \dot{x}(t))$ and the second component $f_{2}=-\ddot{u}_{g}(t)-2 \zeta \omega_{n} \dot{x}(t)-\omega_{n}^{2} x(t)$ of $f$, where $\ddot{u}_{g}(t)$ is a time-sampling record of the acceleration of a ground motion. The barcode is formed by plotting Sign := sign $\left(\|\mathbf{f}\|^{2}\|x\|^{2}-2(f \cdot x)^{2}\right)$ with respect to time. We analyze the complex jumping behavior in a barcode and an essential property of a high percentage occupation of the first set of dis-connectivity in the barcode from four strong earthquake records: 1940 El Centro earthquake, 1989 Loma earthquake, and two records of 1999 Chi-Chi earthquake. Through the comparisons of four earthquakes, we can observe that strong earthquake leads to large percentage of the first set of dis-connectivity.

\section{Keywords}

Earthquakes, Single-Degree-of-Freedom System, Signum Function, Barcode, Jumping Behavior, The First Set of Dis-Connectivity, Scale Invariance

\section{Introduction}

Earthquakes occur around the world frequently. The most are so small that they cannot be susceptible, and only 
a few are large enough to cause a noticeable damage of building. When a structure is subjected to an earthquake motion, its base foundation tends to move with the ground.

One of the most important applications of structural dynamics is by analyzing the response of structures to ground shaking caused by an earthquake, whose data are useful for establishing the structural response spectra. The identification and evaluation of ground motion parameters require access to the measurements of strong ground motions in an actual earthquake [1]-[3].

The basic element of an accelerograph is a transducer element as described in [4] [5], which in its simplest form is a single-degree-of-freedom mass-damper-spring system. Typically, the transducer has natural frequency $f_{n}=25 \mathrm{~Hz}$ and damping ratio $\zeta=60 \%$ for modern analog accelerographs, and $f_{n}=50 \mathrm{~Hz}$ and $\zeta=70 \%$ for modern digit accelerographs [4].

A simple stationary representation of earthquake-induced ground acceleration was proposed by Kanai [6] and Tajimi [7], based on the study of frequency content of a number of strong ground motion records. They suggested that the ground acceleration of the earth surface layer could be approximated by the absolute acceleration of a simple oscillator with a concentrated mass supported by a linear spring and a dashpot. The spectrum property of an earthquake is reflected in its interaction with the structures which have different damping ratios and natural periods. In general, one can use the response spectrum, power spectrum or the Fourier spectrum to characterize the spectrum property of an earthquake. In this paper we propose a novel method to characterize the earthquake property when it is exerted on a simple one-degree-of-freedom system:

$$
\ddot{x}(t)+2 \zeta \omega_{n} \dot{x}(t)+\omega_{n}^{2} x(t)=p(t),
$$

where $p(t)=-\ddot{u}_{g}(t)$ is the ground acceleration due to an earthquake; $\zeta$ is the damping ratio; and $\omega_{n}$ is the natural frequency.

The ground motion produced by earthquake can be very complicated. The exact form of earthquakes is subjected to a high degree of uncertainty and complexity. There are several ways to estimate the ground motion parameters, which are essential for describing the important characteristics of strong ground motion in a compact quantitative form. Because of the complexity of the earthquake ground motion, the identification of a single parameter that can accurately describe all important ground motion characteristics is impossible. In this paper we try to use the barcode and the percentage of the first set of dis-connectivity in the barcode to reflect the characteristic property of an earthquake ground motion. It is known that a barcode is an optical machine-readable representation of data relating to the object to which it is attached. A main feature of the barcode is the intervened black lines and white lines with varying spacings and widths. Barcode is ubiquitously used in the identification and classification of human made products. Here we will use the barcode to identify the property of earthquake with the input $p(t)=-\ddot{u}_{g}(t)$ in Equation (1).

\section{A System Formulation}

To facilitate the formulation we write a system of first-order ordinary differential equations (ODEs):

$$
\dot{\mathbf{x}}=\mathbf{f}(\mathbf{x}, t), \quad t \in \mathrm{R}, \quad \mathbf{x} \in \mathrm{R}^{n} .
$$

Here $n=2, \quad \mathbf{x}=(x, y)=(x, \dot{x})$, and two components of $\mathbf{f}$ are $f_{1}=y$ and $f_{2}=p(t)-\omega_{n}^{2} x-2 \zeta \omega_{n} y$, respectively.

As that done by Liu [8], for Equation (2) we can define a unit orientation vector:

$$
\mathbf{n}:=\frac{\mathbf{x}}{\|\mathbf{x}\|},
$$

where $\|\mathbf{x}\|:=\sqrt{\mathbf{x} \cdot \mathbf{x}}>0$ is the Euclidean norm of $\mathbf{x}$, and the dot between two vectors, say $\mathbf{x} \cdot \mathbf{y}$, denotes the inner product of $\mathbf{x}$ and $\mathbf{y}$. Then we can derive [9]-[11]

$$
\begin{gathered}
\dot{\mathbf{x}}=\frac{\mathbf{f} \cdot \mathbf{x}}{\|\mathbf{x}\|^{2}} \mathbf{x}+\left[\frac{\mathbf{f}}{\|\mathbf{x}\|} \otimes \frac{\mathbf{x}}{\|\mathbf{x}\|}-\frac{\mathbf{x}}{\|\mathbf{x}\|} \otimes \frac{\mathbf{f}}{\|\mathbf{x}\|}\right] \mathbf{x}, \\
\frac{\mathrm{d}}{\mathrm{d} t}\|\mathbf{x}\|=\mathbf{f} \cdot \frac{\mathbf{x}}{\|\mathbf{x}\|} .
\end{gathered}
$$


Equations (4) and (5) can be put together as

$$
\frac{\mathrm{d}}{\mathrm{d} t}\left[\begin{array}{c}
\mathbf{x} \\
\|\mathbf{x}\|
\end{array}\right]=\left[\begin{array}{cc}
\frac{\mathbf{f}}{\|\mathbf{x}\|} \otimes^{\otimes} \frac{\mathbf{x}}{\|\mathbf{x}\|}-\frac{\mathbf{x}}{\|\mathbf{x}\|} \otimes \frac{\mathbf{f}}{\|\mathbf{x}\|} & \frac{(\mathbf{f} \cdot \mathbf{x}) \mathbf{x}}{\|\mathbf{x}\|^{3}} \\
\frac{(\mathbf{f} \cdot \mathbf{x}) \mathbf{x}^{\mathrm{T}}}{\|\mathbf{x}\|^{3}} & 0
\end{array}\right]\left[\begin{array}{c}
\mathbf{x} \\
\|\mathbf{x}\|
\end{array}\right],
$$

which is a Lie-type system endowing with the Lie-group symmetry $S O_{o}(n, 1)$ in the Minkowski space.

\section{Two Branches Solutions}

In order to develop a numerical scheme from Equation (6), we suppose that the coefficient matrix is constant with the pair

$$
(\mathbf{a}, \mathbf{b})=\left(\frac{\mathbf{f}}{\|\mathbf{x}\|}, \frac{\mathbf{x}}{\|\mathbf{x}\|}\right)
$$

being constant in a small time stepsize.

From Equations (6) and (7) we thus need to solve a constant linear system:

$$
\frac{\mathrm{d}}{\mathrm{d} t}\left[\begin{array}{c}
\mathbf{x} \\
\|\mathbf{x}\|
\end{array}\right]=\left[\begin{array}{cc}
\mathbf{a} \otimes \mathbf{b}-\mathbf{b} \otimes \mathbf{a} & \mathbf{a} \cdot \mathbf{b} \mathbf{b} \\
\mathbf{a} \cdot \mathbf{b} \mathbf{b}^{\mathrm{T}} & 0
\end{array}\right]\left[\begin{array}{c}
\mathbf{x} \\
\|\mathbf{x}\|
\end{array}\right] .
$$

Let

$$
\begin{aligned}
z=\mathbf{a} \cdot \mathbf{x}, \quad w & =\mathbf{b} \cdot \mathbf{x}, \quad y=\|\mathbf{x}\|, \\
c_{0} & =\mathbf{a} \cdot \mathbf{b},
\end{aligned}
$$

and Equation (8) becomes

$$
\begin{gathered}
\dot{\mathbf{x}}=w \mathbf{a}-z \mathbf{b}+c_{0} y \mathbf{b}, \\
\dot{y}=c_{0} w .
\end{gathered}
$$

At the same time, from the above equations we can derive the following ODEs for $z, w$ and $y$ :

$$
\frac{\mathrm{d}}{\mathrm{d} t}\left(\begin{array}{c}
\mathrm{z} \\
w \\
y
\end{array}\right)=\left(\begin{array}{ccc}
-c_{0} & a_{0}^{2} & c_{0}^{2} \\
-1 & c_{0} & c_{0} \\
0 & c_{0} & 0
\end{array}\right)\left[\begin{array}{c}
\mathrm{z} \\
w \\
y
\end{array}\right],
$$

where $a_{0}=\|\mathbf{a}\|$. Fortunately, the original $(n+1)$-dimensional problem in Equation (8) can be reduced to a threedimensional problem in Equation (13). Depending on the signum function of

$$
\operatorname{Sign}:=\operatorname{sign}\left(a_{0}^{2}-2 c_{0}^{2}\right)=\operatorname{sign}\left(\|\mathbf{x}\|^{2}\|\mathbf{f}\|^{2}-2(\mathbf{f} \cdot \mathbf{x})^{2}\right),
$$

there exist two different types solutions of $(z, w, y)$. Here, we do not give a detailed derivation of the solutions for $(z, w, y)$ and the corresponding second generation group preserving scheme (GPS2), but the reader can refer [9]-[11].

\section{The Demonstration of Barcodes}

It is significant that in Equation (14) we have derived a signum function to demand the algorithm into two branches. Without having the factor 2 before $\mathbf{f} \cdot \mathbf{x}$ in Equation (14) one has

$$
\|\mathbf{f}\|^{2}\|\mathbf{x}\|^{2}-(\mathbf{f} \cdot \mathbf{x})^{2}=\|\mathbf{f}\|^{2}\|\mathbf{x}\|^{2}\left(1-\cos ^{2} \theta\right) \geq 0,
$$

where $\theta$ is the intersection angle between $\mathbf{x}$ and $\mathbf{f}$; hence, it makes no sense to define its signum function. On the contrast, by Equation (14) we have 


$$
\text { Sign }=\operatorname{sign}\left(\|\mathbf{f}\|^{2}\|\mathbf{x}\|^{2}\left(1-2 \cos ^{2} \theta\right)\right)=-\operatorname{sign}(\cos 2 \theta),
$$

which may be +1 or -1 , depending on $\mathbf{x}$ and $\mathbf{f}$ by their intersection angle $\theta$. When $\theta$ is in the range of $-\pi / 2<\theta<\pi / 2$ or $3 \pi / 4<\theta<5 \pi / 4$, the value of Sign is Sign $=-1$; otherwise, Sign $=+1$. Thus we can observe the time-varying values of Sign and plot them as a barcode. In order to demonstrate the use of barcode, let us investigate the following examples. In all computations given below the initial conditions are set to be $(x(0), \dot{x}(0))=(0,0)$, unless specifying otherwise.

\subsection{Example 1}

First we test an example with $\omega_{n}=\sqrt{2}$ and $\zeta=0.15 / \sqrt{2}$, and a harmonic force $p(t)=$ cost is applied to Equation (1) in a time interval of $t \in[0,100]$. The input, the following signum function:

$$
\operatorname{Sign}(t)=\operatorname{sign}\left\{f_{1}^{2}(t)+f_{2}^{2}(t)+x^{2}(t)+y^{2}(t)-2\left[x(t) f_{1}(t)+y(t) f_{2}(t)\right]^{2}\right\},
$$

where $f_{1}(t)=y(t)$ and $f_{2}(t)=p(t)-\omega_{n}^{2} x(t)-2 \zeta \omega_{n} y(t)$, and the responses of $x(t)$ and $y(t)$ are plotted in Figure 1. It is interesting that after $0.78 \mathrm{sec}$, Sign $=+1$ for the harmonic input. As compared with the closedform solution, in Figure 1(d) we plot the numerical error, which is quite accurate, and is smaller than the time stepsize $h=0.001$ used in this computation.
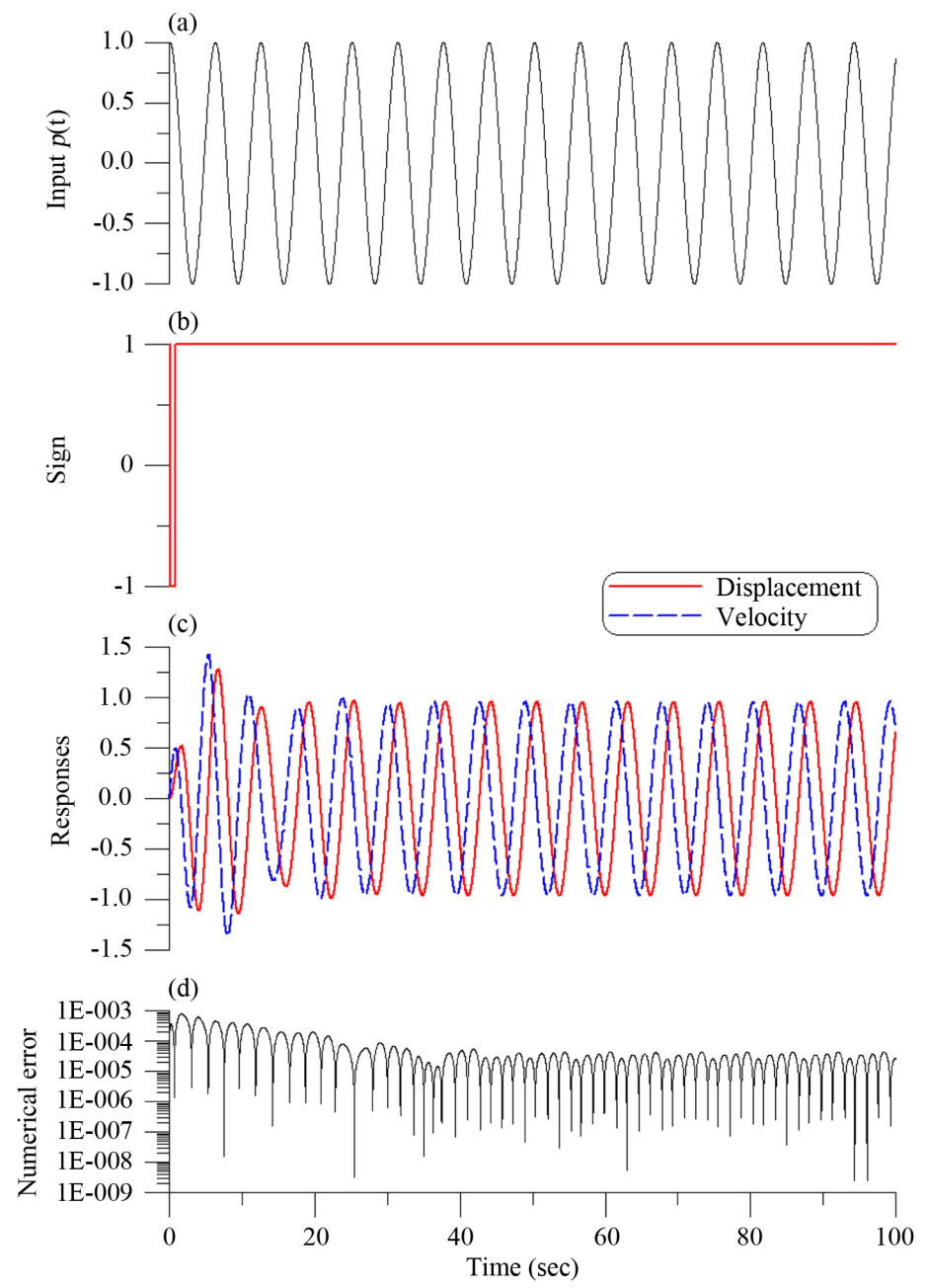

Figure 1. Under (a) a harmonic force, showing (b) the signum function, (c) the responses, and (d) numerical error of displacement. 


\subsection{Example 2}

Then with $\omega_{n}=\sqrt{3}$ and $\zeta=0.1 / \sqrt{3}$, and under a random force

$$
p\left(t_{i}\right)=5 R_{i},
$$

where $R_{i}$ are random numbers between $[-1,1]$, the input, the Sign and the responses are plotted in Figure 2. It is interesting that the values of Sign are randomly jumping between +1 and -1 in some intervals of time.

\subsection{Analyzing the Barcode under Earthquakes}

With $\omega_{n}=2 \pi / 1.5$ and $\zeta=0.02$, and under the El Centro earthquake $\ddot{u}_{g}(t)$, which is adjusted to $p(t)=-0.98 \ddot{u}_{g}(t) \mathrm{m} / \mathrm{sec}^{2}$ as the input to Equation (1), the barcode and the responses are plotted in Figure 3. As shown in Figure 3(b), the time history of Sign looks like a barcode, which is interesting that the values of Sign are frequently jumping between +1 and -1 with a certain structure.

Now we analyze the Sign and the length as shown in Figure 4 within a short time interval of $t \in[0,2]$, which is starting from the initial conditions $(x(0), \dot{x}(0))=(0.1,0.01)$, where we take $\omega_{n}=\sqrt{2}$ and $\zeta=0.01 / \sqrt{2}$ and $p(t)=-50 \ddot{u}_{g}(t)$ being the input. First we note that the length as governed by Equation (5) has the following property:
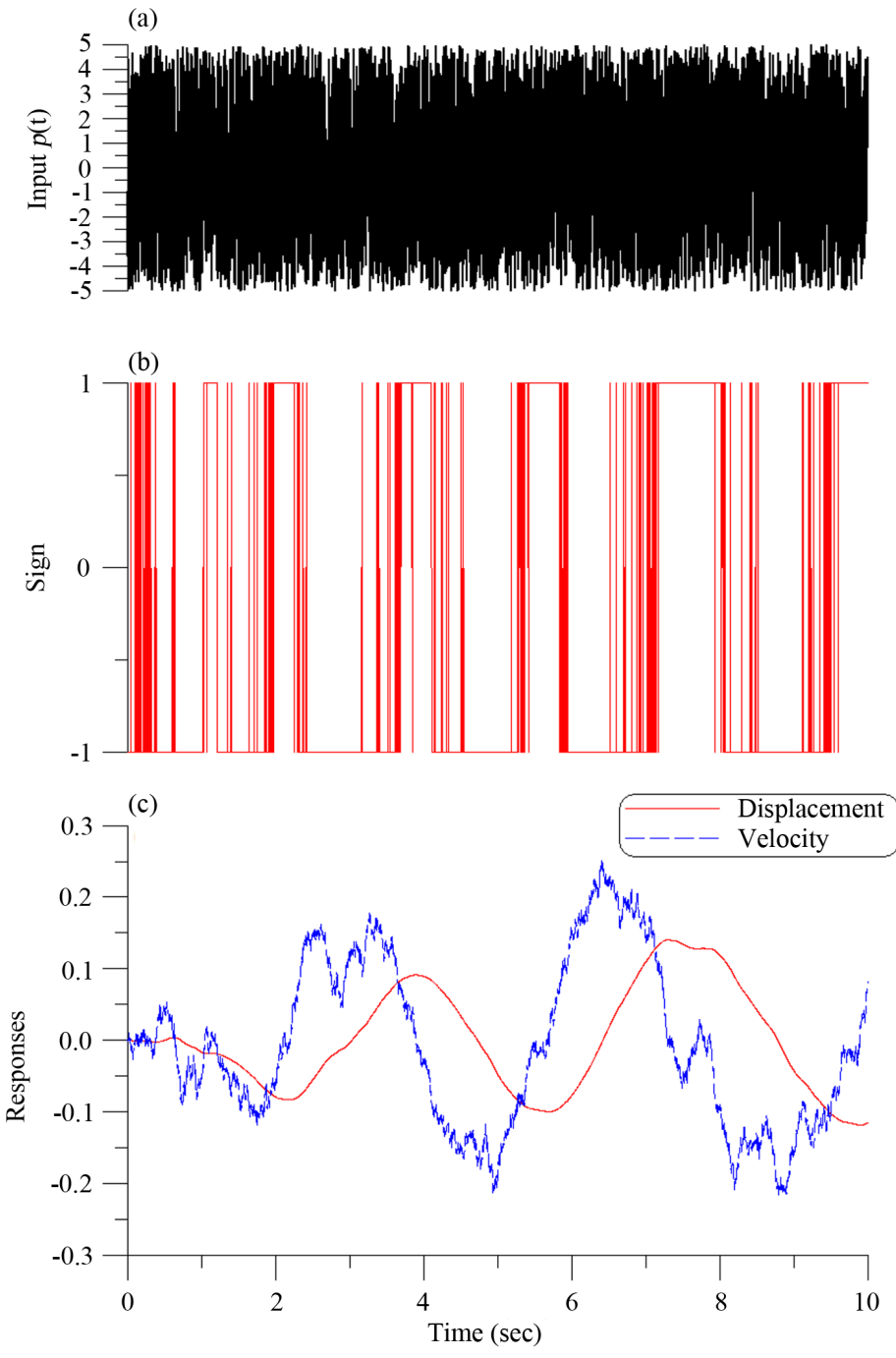

Figure 2. Under (a) a random force, showing (b) the signum function, and (c) the responses. 

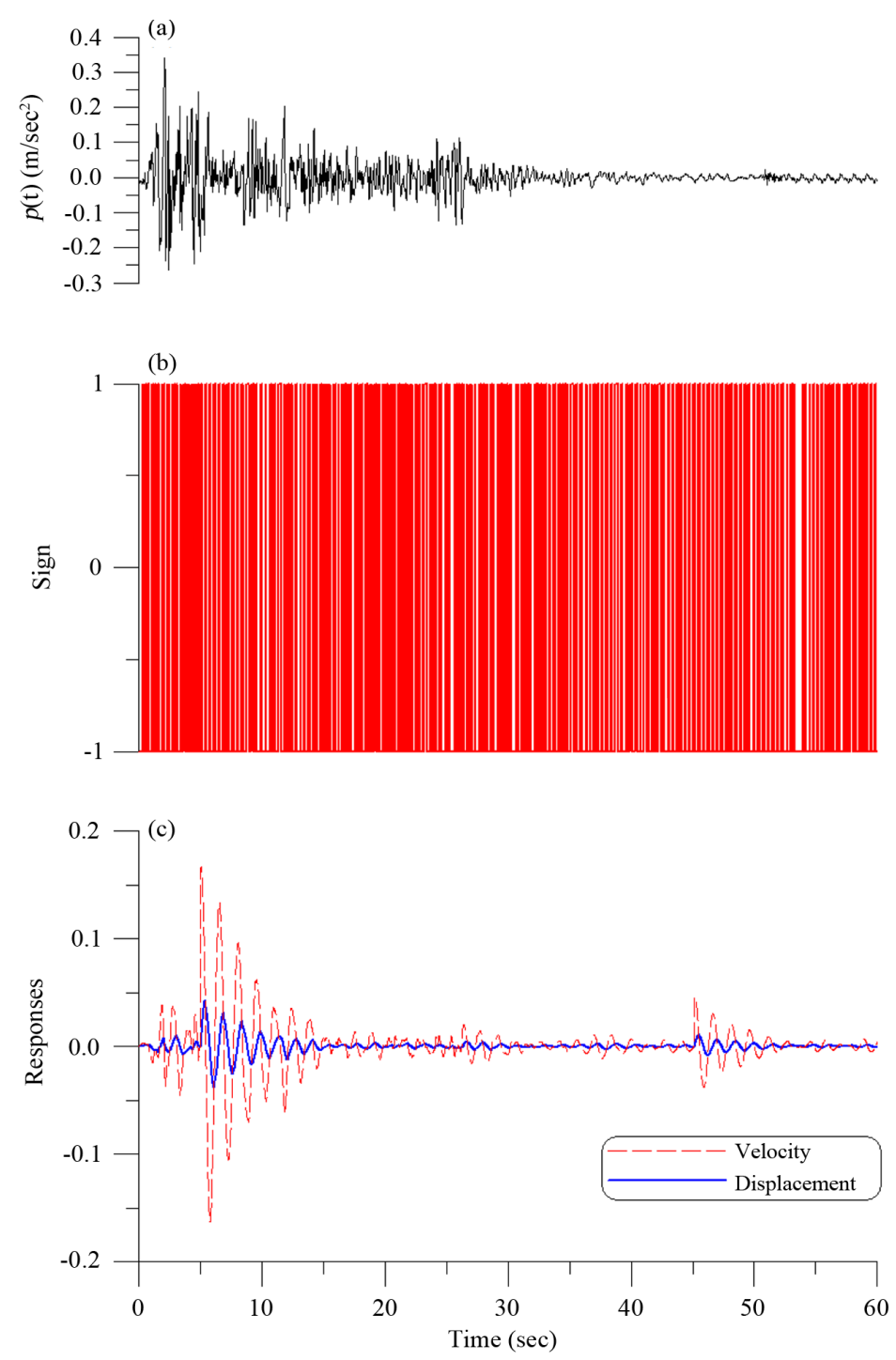

Figure 3. Under (a) the El Centro earthquake, showing (b) the barcode, and (c) the responses.

$$
\operatorname{sign}\left(c_{0}\right) \stackrel{<}{>} 0 \Leftrightarrow \frac{\mathrm{d}}{\mathrm{d} t}\|\mathbf{x}\|_{>}^{<} 0
$$

where $\operatorname{sign}\left(c_{0}\right)=\operatorname{sign}(\mathbf{f} \cdot \mathbf{x})$. Then, as a schematic plot shown in Figure 5, there are two dis-connected sets of $\|\mathbf{f}\|^{2}\|\mathbf{x}\|^{2}-2(\mathbf{f} \cdot \mathbf{x})^{2}<0$ in the hyper-plane $(\mathbf{x}, \mathbf{f})$ :

$$
\begin{gathered}
\mathbf{f} \cdot \mathbf{x}>\frac{1}{\sqrt{2}}\|\mathbf{f}\|\|\mathbf{x}\|, \\
\mathbf{f} \cdot \mathbf{x}<-\frac{1}{\sqrt{2}}\|\mathbf{f}\|\|\mathbf{x}\| .
\end{gathered}
$$

We may call them the first set of dis-connectivity and the second set of dis-connectivity, respectively. Clearly, the first set of dis-connectivity is a subset of $\operatorname{sign}\left(c_{0}\right)=+1$, and the second set of dis-connectivity is a subset of $\operatorname{sign}\left(c_{0}\right)=-1$.

If Equation (2) satisfies 

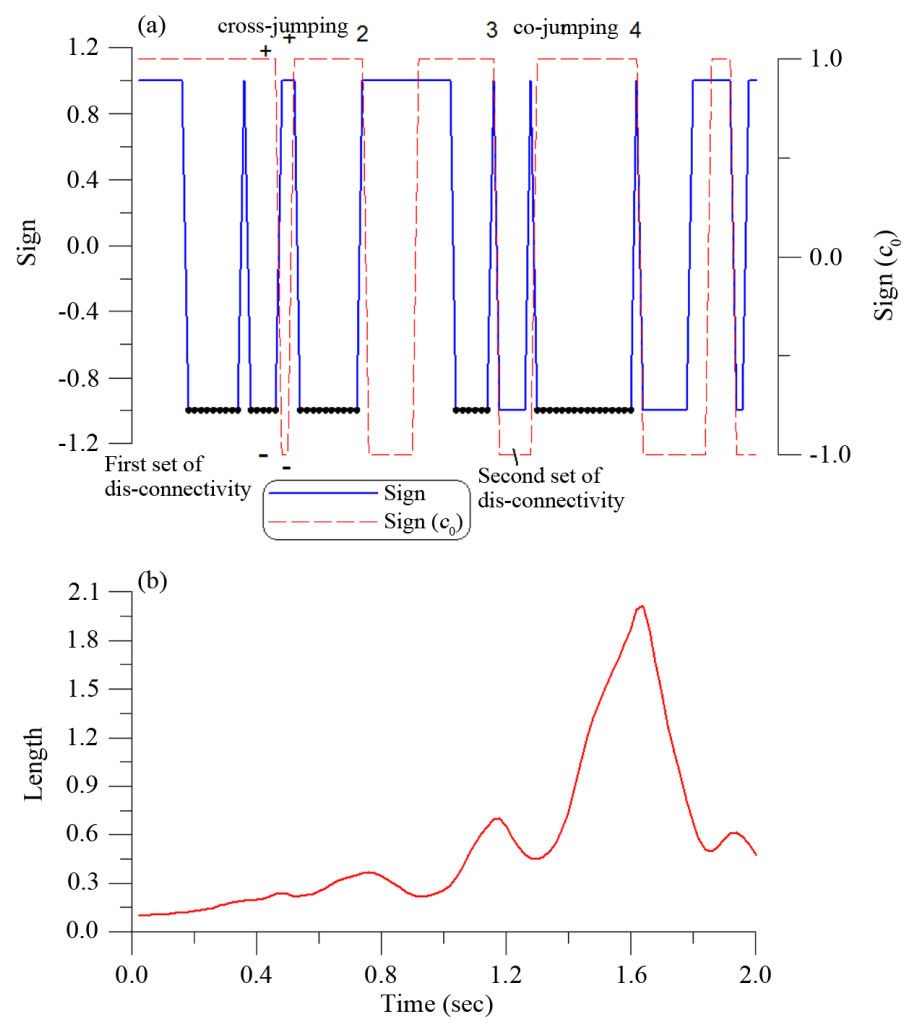

Figure 4. Under the El Centro earthquake, (a) showing two signum functions, and (b) the length with a small time interval.

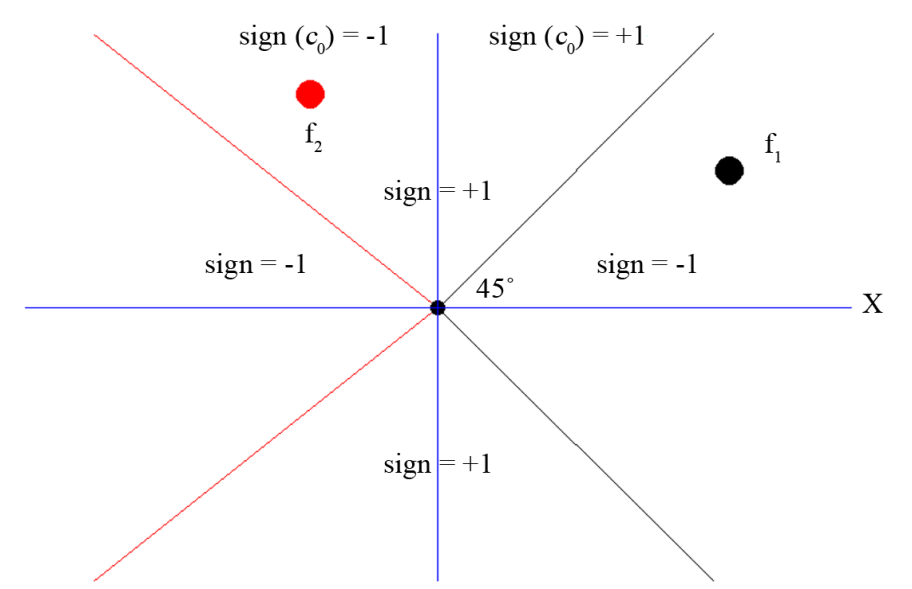

Figure 5. A schematic plot of the area of Sign $=+1$, Sign $=-1$, $\operatorname{sign}\left(c_{0}\right)=$ +1 , and $\operatorname{sign}\left(c_{0}\right)=-1$ in the plane.

$$
\mathbf{f}\left(\mathbf{x}\left(t_{0}\right), t_{0}\right) \cdot \mathbf{x}\left(t_{0}\right)>0 \text {, and } \operatorname{sign}\left(\|\mathbf{f}\|^{2}\|\mathbf{x}\|^{2}-2(\mathbf{f} \cdot \mathbf{x})^{2}\right)=-1, \quad \forall t \geq t_{0} \text {, then } \mathbf{x}(t) \rightarrow \infty, t \rightarrow \infty .
$$

Under the first assumption the case in Equation (20) is impossible because it contradicts to $\mathbf{f}\left(\mathbf{x}\left(t_{0}\right), t_{0}\right) \cdot \mathbf{x}\left(t_{0}\right)>0$. Then under the condition of $\operatorname{sign}\left(\|\mathbf{f}\|^{2}\|\mathbf{x}\|^{2}-2(\mathbf{f} \cdot \mathbf{x})^{2}\right)=-1$, it is always

$$
\mathbf{f} \cdot \mathbf{x}>\frac{1}{\sqrt{2}}\|\mathbf{f}\|\|\mathbf{x}\|, \quad \forall t \geq t_{0},
$$


because the two sets of dis-connectivity are dis-connected, and from the first set to the second set it must go to $\operatorname{sign}\left(\|\mathbf{f}\|^{2}\|\mathbf{x}\|^{2}-2(\mathbf{f} \cdot \mathbf{x})^{2}\right)=+1$ in some time interval. Then using Equations (5) and (22) we have

$$
\frac{\mathrm{d}}{\mathrm{d} t}\|\mathbf{x}\|>\frac{1}{\sqrt{2}}\|\mathbf{f}\|>0
$$

which means that the length grows with time. Thus, Equation (21) is proven.

For a system being excited by earthquake the value of Sign is not always +1 as that shown in Figure 1(b) for the harmonic input. As shown in Figure 3(b) the values of Sign are varying between -1 to +1 and +1 to -1 very fast, and then the Sign will return to +1 again as shown in Figure 4(a); otherwise, by Equation (21) the system will respond unstably and thus the displacement will tend to infinity. In order to compare the values of $\operatorname{sign}\left(c_{0}\right)$ with the values of Sign we plot them in Figure 4(a) with solid line and dashed line, respectively, where we make a slight shift of the dashed line downward for clear. On the other hand, for a distinction the first set of dis-connectivity is filled by solid black points.

As shown in Figure 5, in the plane the areas of $\operatorname{sign}\left(c_{0}\right)=+1$ and $\operatorname{sign}\left(c_{0}\right)=-1$ are connected while that the sets of Sign $=+1$ and Sign $=-1$ are dis-connected as mentioned in the above. When $\operatorname{sign}\left(c_{0}\right)$ changes from +1 to -1 , for example from point $\mathbf{f}_{1}$ to point $\mathbf{f}_{2}$ in the figure, the Sign must jump from -1 to +1 . This jumping behavior is termed a cross jumping. On the other hand, we also find the co-jumping behavior which is happened when both the Sign and $\operatorname{sign}\left(c_{0}\right)$ are jumping from -1 to +1 simultaneously. The above two jumping behaviors are collected as a coupled-jumping, which renders a quite complex structure of the barcode as shown in Figure 3(b).

From Figure 4(a) we can observe the following interesting phenomena: 1) While the first set of dis-connectivity is a subset of $\operatorname{sign}\left(c_{0}\right)=+1$, the second set of dis-connectivity is a subset of $\operatorname{sign}\left(c_{0}\right)=-1$. 2) In a time interval of the state with Sign $=-1$, the state is either in the first set of dis-connectivity or in the second set of disconnectivity. 3) From one first set of dis-connectivity to another first set of dis-connectivity there must accompany a jump from Sign $=-1$ to Sign $=+1$. This also holds for the second set of dis-connectivity. 4) From one first set of dis-connectivity to one second set of dis-connectivity, or vice-versa, there must accompany a jump from Sign $=-1$ to Sign $=+1$. For some cases this jumping only happens at one time point. 5) When $\operatorname{sign}\left(c_{0}\right)$ jumps from +1 to -1 as remarked in Figure 4 (a) by the symbols + and - (i.e., the length is decreased), and if the Sign is in the state of -1 , then the Sign will jump from -1 to +1 as remarked in Figure 4(a) by the symbols and + . Indeed in Figure 4(a) we can observe that there are four times to happen the cross jumping behavior in the interval of $t \in[0,2]$. Besides the first one, the other three times are marked by the Arabic numbers 2, 3 and 4 in Figure 4(a). Besides, there is also a co-jumping as remarked in Figure 4(a). The proof of 5) is obvious by viewing Figure 5 that the first set of Sign $=-1$ cannot directly jump to the second set of Sign $=-1$. When $\mathbf{f}_{1}$ goes to $\mathbf{f}_{2}$, the Sign changes from -1 to +1 .

In Figure 6 we compare the barcodes with 1) $\omega_{n}=2 \pi / 1.5, \zeta=0.02$ and $\left.p(t)=-0.98 \ddot{u}_{g}(t) \mathrm{m} / \mathrm{sec}^{2}, 2\right)$ $\omega_{n}=2 \pi / 1.5, \zeta=0.02$ and $\left.p(t)=-4.9 \ddot{u}_{g}(t) \mathrm{m} / \mathrm{sec}^{2}, 3\right) \omega_{n}=2 \pi / 1.5, \zeta=0.05$ and $p(t)=-0.98 \ddot{u}_{g}(t) \mathrm{m} / \mathrm{sec}^{2}$, and 4) $\omega_{n}=2 \pi / 2, \quad \zeta=0.02$ and $p(t)=-0.98 \ddot{u}_{g}(t) \mathrm{m} / \mathrm{sec}^{2}$.

\subsection{The First Set of Dis-Connectivity under Earthquakes}

In this example we let the Chi-Chi earthquake be the input to Equation (1). With $\omega_{n}=2 \pi / 1.5$ and $\zeta=0.02$, and under the Chi-Chi earthquake $\ddot{u}_{g}(t)$ recorded at the station of CHY028, we adjust it to be $p(t)=-0.98 \ddot{u}_{g}(t) \mathrm{m} / \mathrm{sec}^{2}$ as shown in Figure 7(a). The barcode and the responses are plotted in Figure 7(b) and Figure 7(c).

In Figure 8 we compare the barcodes with 1) $\omega_{n}=2 \pi / 1.5, \zeta=0.02$ and $\left.p(t)=-0.98 \ddot{u}_{g}(t) \mathrm{m} / \mathrm{sec}^{2}, 2\right)$ $\omega_{n}=2 \pi / 1.5, \zeta=0.02$ and $\left.p(t)=-4.9 \ddot{u}_{g}(t) \mathrm{m} / \mathrm{sec}^{2}, 3\right) \omega_{n}=2 \pi / 1.5, \zeta=0.05$ and $p(t)=-0.98 \ddot{u}_{g}(t) \mathrm{m} / \mathrm{sec}^{2}$, and 4) $\omega_{n}=2 \pi / 0.5, \zeta=0.02$ and $p(t)=-0.98 \ddot{u}_{g}(t) \mathrm{m} / \mathrm{sec}^{2}$.

In Figure 9(a) for the Chi-Chi earthquake with different scaling factor $f_{r}$ in $p(t)=-9.8 f_{r} \ddot{u}_{g}(t)$ as being the input to Equation (1), and with $\omega_{n}=2 \pi / 1.5$ and $\zeta=0.02$, we plot the percentage in the first set of dis-connectivity in Figure 9(b), which is near to forty eight percentage, and at the same time we also display the numbers 
(a)

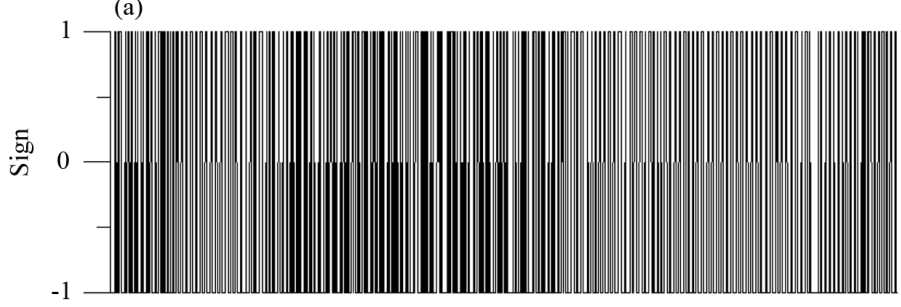

(b)

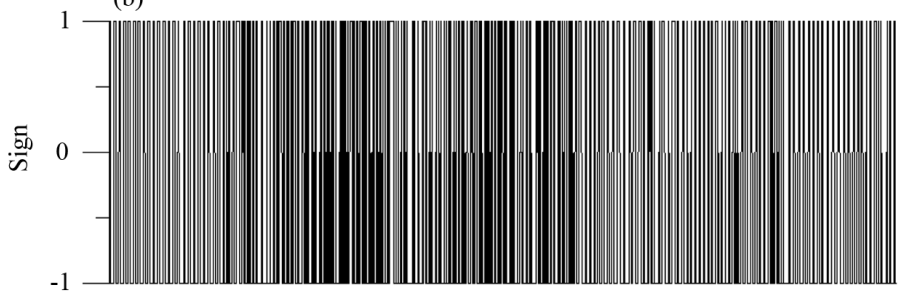

(c)

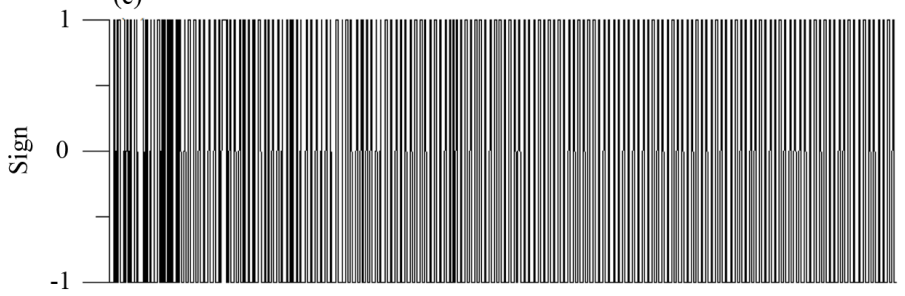

(d)

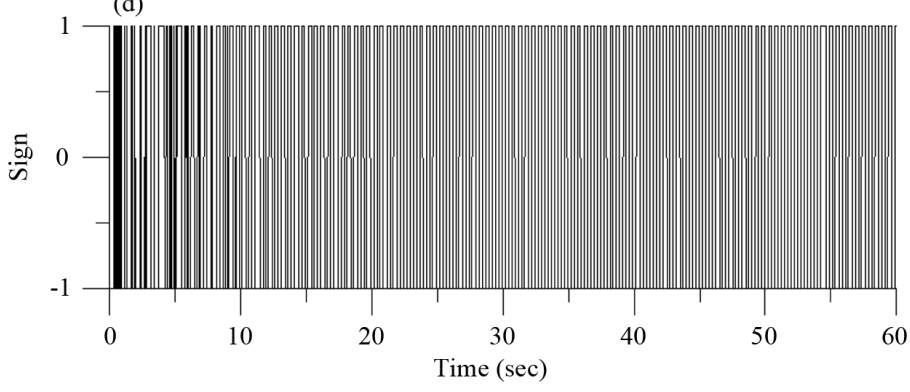

Figure 6. Under the El Centro earthquake, comparing the barcodes under different scaling factor, damping ratio and natural period.

of coupled-jumping as discussed above with respect to the scaling factor $f_{r}$ in Figure 9(c). It can be seen that the number is either 24 or 25. In the plane as shown in Figure 5, the first set of dis-connectivity with Sign $=-1$ is only in one quarter. But under a strong earthquake, the high occupation of the first set of dis-connectivity is near to fifty percentage as shown in Figure 9(b).

In Figure 10, under the Chi-Chi earthquake (CHY080) as shown in (a), we plot the first set of dis-connectivity which is near to forty nine percentage as shown in Figure 10(b), and the number of coupled-jumping is plotted in Figure 10(c), which is either 6 or 7.

In Figure 11(a) for the El Centro earthquake (ELC180) with different scaling factor $f_{r}$ in $p(t)=-9.8 f_{r} \ddot{u}_{g}(t)$, and with $\omega_{n}=\pi$ and $\zeta=0.02$, we plot the first set of dis-connectivity which is near to forty six percentage as shown in Figure 11(b), and the number of coupled-jumping is plotted in Figure 11(c), which is 0 or 1 for most scaling factors. In Figure 12(a) for the Loma Prieta earthquake (47379 Gilroy Array \# 1) with different scaling factor $f_{r}$ in $p(t)=-9.8 f_{r} \ddot{u}_{g}(t)$, and with $\omega_{n}=\pi$ and $\zeta=0.02$, we plot the first set of dis-connectivity which is near to forty six percentage as shown in Figure 12(b), and the number of coupled-jumping is plotted in Figure 12(c), which is between 1 to 7 .

It is surprising that the percentage of the first set of disconnectivity and the number of coupled-jumping are almost constant for each earthquake, which seems to be scale-invariant. In the plane as shown in Figure 5, the 

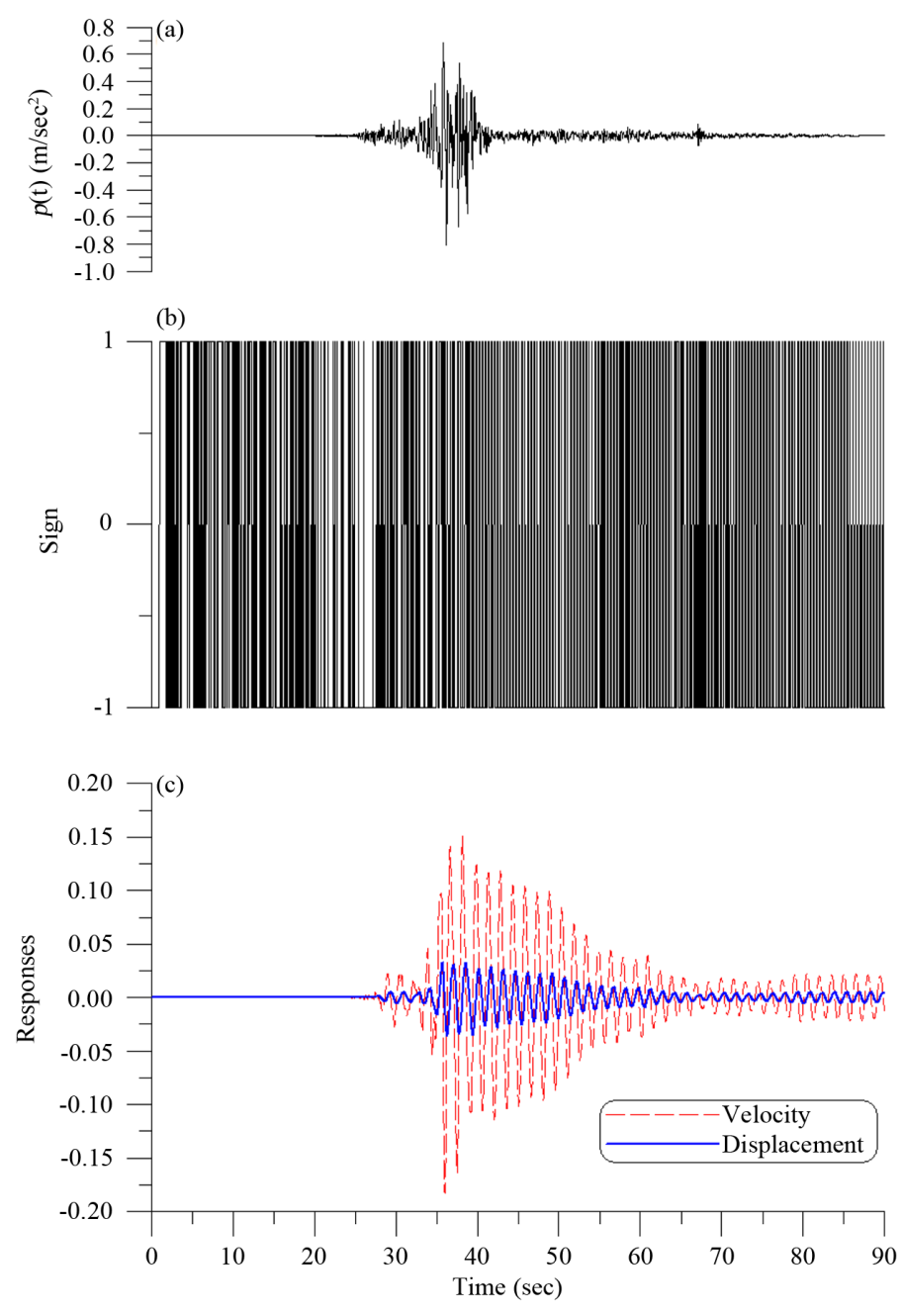

Figure 7. Under the Chi-Chi earthquake (CHY028) in (a), (b) showing the barcode, and (c) the responses.

first set of dis-connectivity with Sign $=-1$ is only in one quarter. But under strong earthquakes, the high occupation of the first set of dis-connectivity is near to fifty percentage. The high percentage of occupation demonstrates the importance of the first set of dis-connectivity in the strong earthquake motion. As comparing the above four earthquakes we can also observe that stronger earthquake leads to larger percentage of the first set of dis-connectivity. Finally, under the same scaling factor $f_{r}=0.5$ in $p(t)=-9.8 f_{r} \ddot{u}_{g}(t)$ and the same $\omega_{n}=\pi$ and $\zeta=0.02$, in Figure 13 we compare the relative percentages of first set of dis-connectivity for the El Centro earthquake (ELC180), the Loma Prieta earthquake (47379 Gilroy Array \#1), and two records (CHY028, CHY080) of Chi-Chi earthquake. The relative percentage is a time history of the time-duration of the first set of dis-connectivity, whose time is dividing by the total time of earthquake. This figure reflects that the Chi-Chi earthquake has larger values than that of the El Centro and the Loma Prieta earthquakes, and CHY080 is larger than CHY028. This can also be explained by calculating the power of earthquake with the $\ell_{2}$-norm of earthquake, of which the power value of CHY080 is 0.7765, of CHY028 is 0.6193 and of the El Centro earthquake is 0.3444. The Loma Prieta earthquake (47379 Gilroy Array \#1) leads to a close curve with that produced by the El Centro earthquake, whose power is 0.33 .

\section{Conclusions}

For a single-degree-of-freedom system subjected to an earthquake excitation, we have investigated the response 
(a)

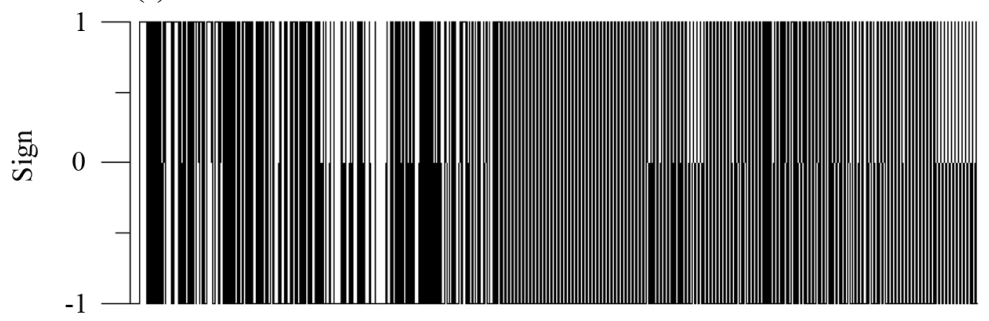

(b)

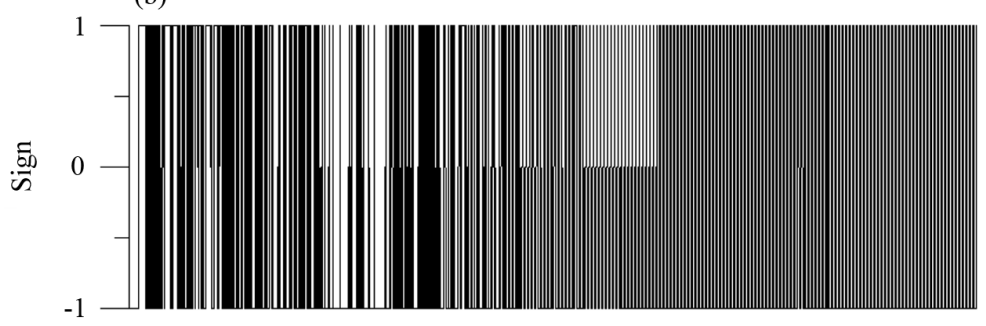

(c)

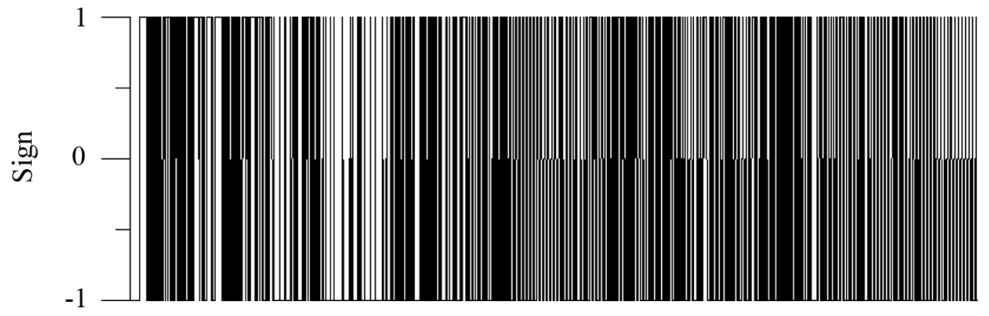

(d)

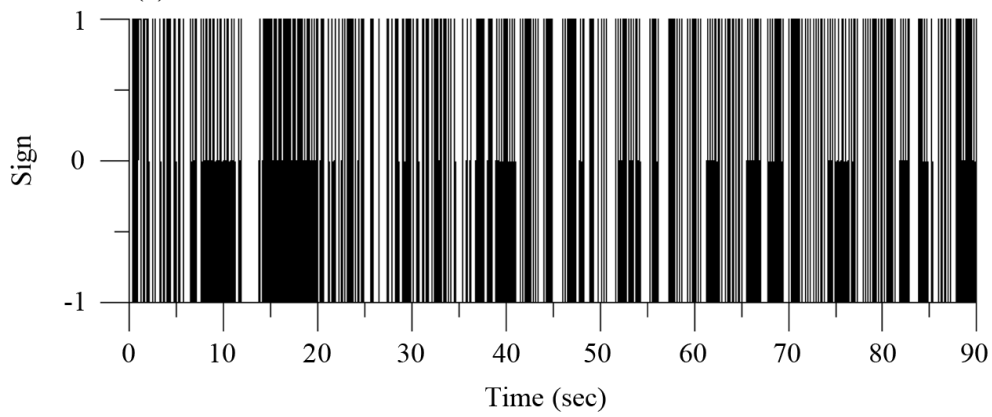

Figure 8. Under the Chi-Chi earthquake (CHY028), comparing the barcodes under different scaling factor, damping ratio and natural period.

behavior from a newly developed theory about the dynamical system of $\dot{\mathbf{x}}=\mathbf{f}(\mathbf{x}, t)$ by viewing the signum function of $\|\mathbf{f}\|^{2}\|\mathbf{x}\|^{2}-2(\mathbf{f} \cdot \mathbf{x})^{2}$. The main idea in this paper is that the earthquake will make the function $\mathbf{f}$ highly complex in time, even the linear system is simple in $\mathbf{x}$. Under this situation, we expect that the signum function being a time varying function will disclose the complexity of earthquake, such that we can study its structure. The barcode was formed by plotting the signum function Sign with respect to the time history of an earthquake. We have analyzed the jumping behavior from two famous earthquake inputs of 1940 El Centro earthquake and 1999 Chi-Chi earthquake. Several barcodes were used to reveal a quite complex structure of earthquake, and we have demonstrated the importance of the first set of dis-connectivity, whose high percentage occupation is very interesting when one considers the linear system under a strong earthquake. Through the comparisons of four earthquake inputs, we have observed that stronger earthquake leads to larger percentage of the first set of dis-connectivity. The coupled-jumping behavior may happen due to the random property of 

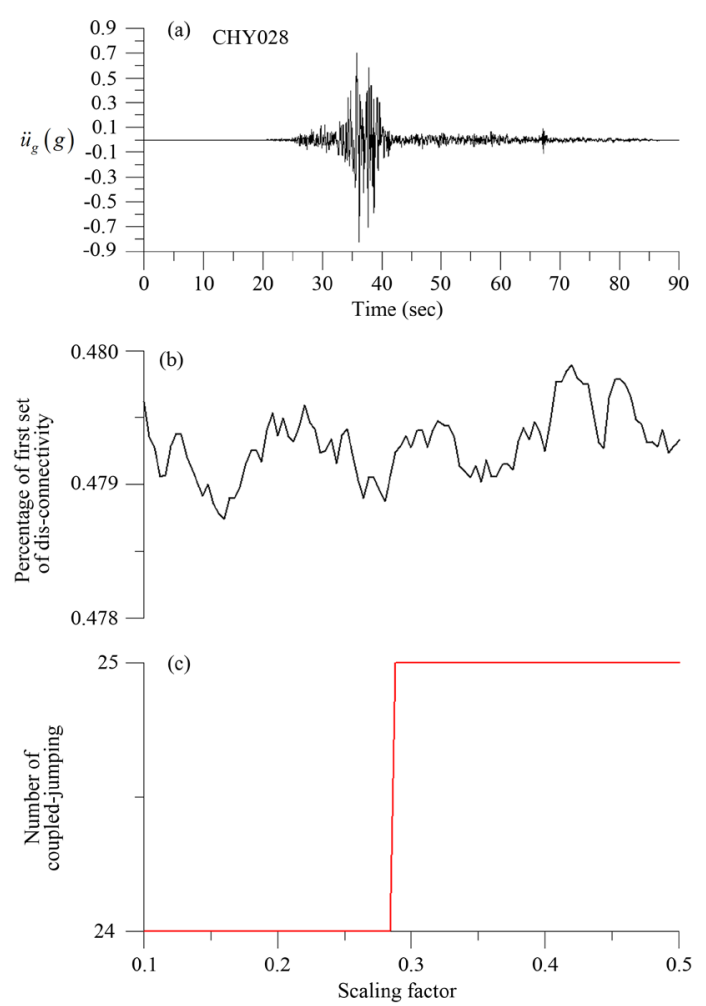

Figure 9. Under (a) the Chi-Chi earthquake (CHY028) with different scaling factor, showing (b) the percentage in the first set of dis-connectivity, and (c) the number of coupled-jumping.
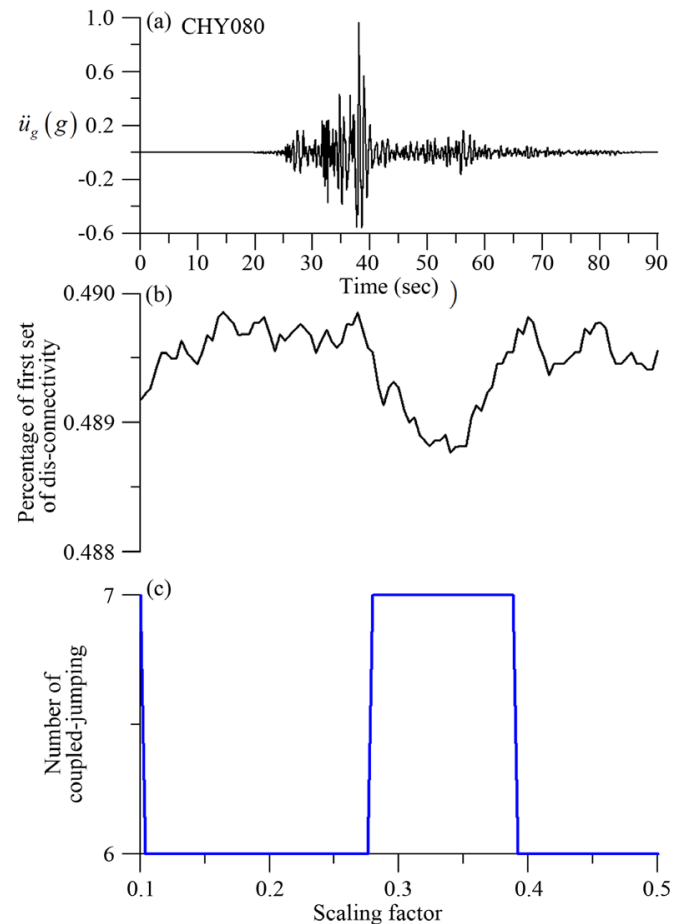

Figure 10. Under (a) the Chi-Chi earthquake (CHY080) with different scaling factor, showing (b) the percentage in the first set of dis-connectivity, and (c) the number of coupled-jumping. 

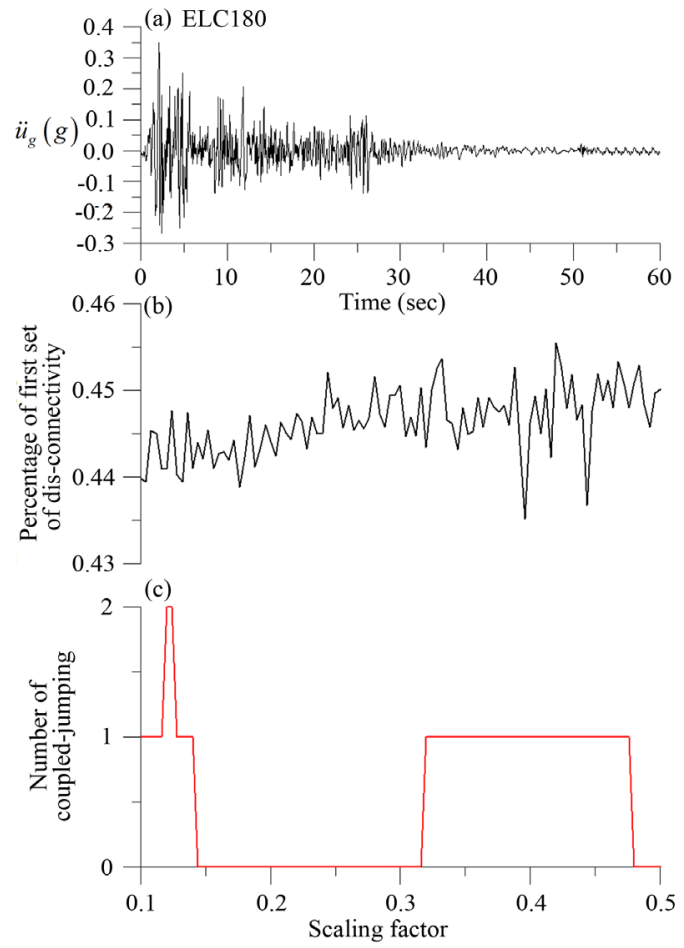

Figure 11. Under (a) the El Centro earthquake (ELC180) with different scaling factor, showing (b) the percentage in the first set of dis-connectivity, and (c) the number of coupled-jumping.
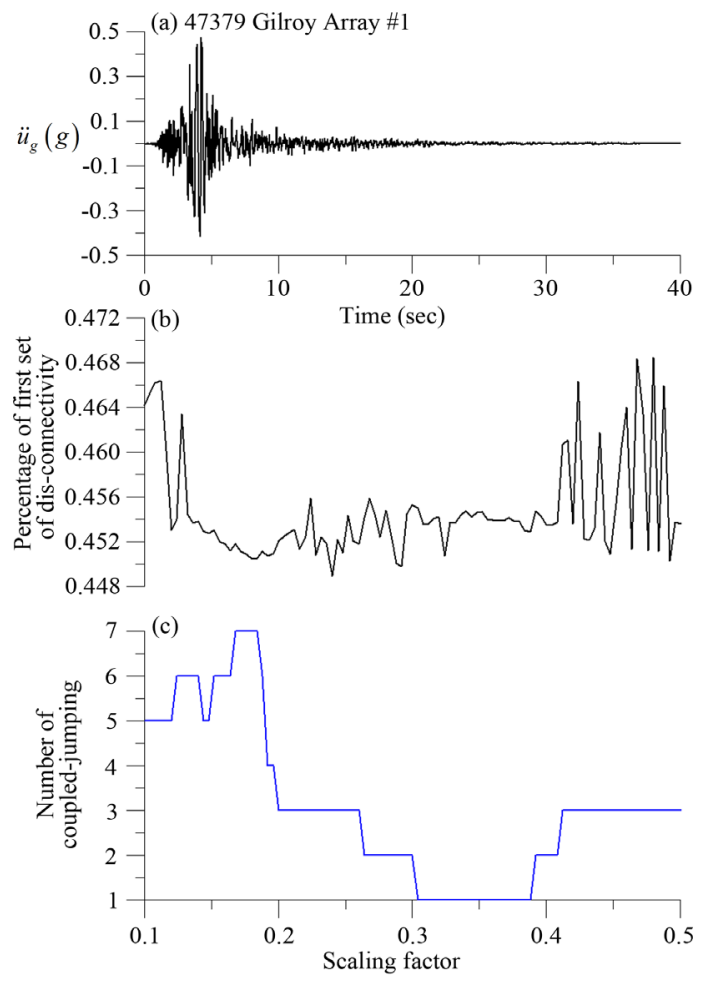

Figure 12. Under (a) the 1989 Loma Prieta earthquake (47379 Gilroy Array \#1) with different scaling factor, showing (b) the percentage in the first set of dis-connectivity, and (c) the number of coupled-jumping. 


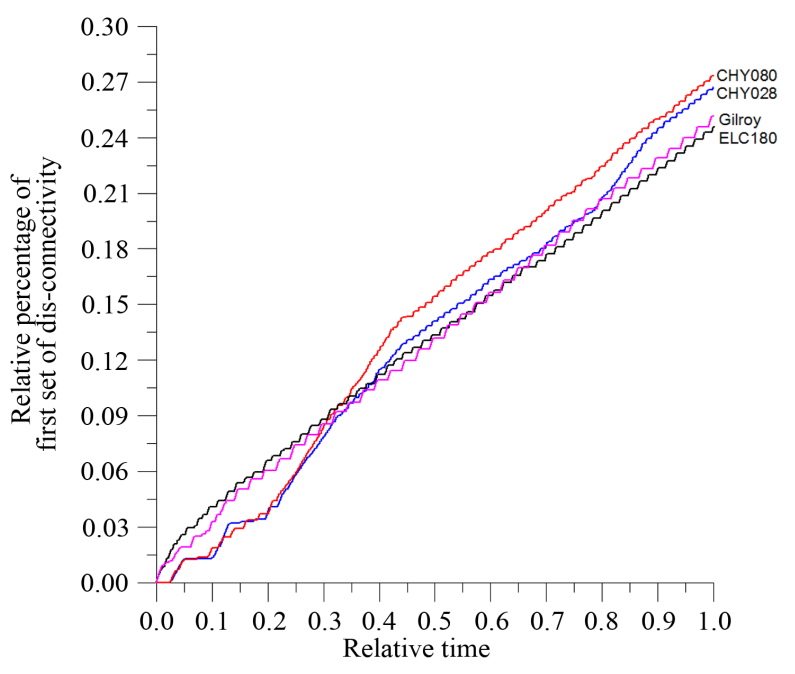

Figure 13. Comparing the relative percentages of first set of dis-connectivity for the El Centro earthquake (ELC180), two records (CHY028, CHY080) of Chi-Chi earthquake, and the Loma Prieta earthquake (47379 Gilroy Array \#1).

earthquakes, and its number seems scale-independence. This is just an initial study by correlating the percentage of the first set of dis-connectivity to the earthquake, and there are still many works to study the barcode and the use of it to forecast the intensity of earthquake before the rapid coming of main shock impulse. In the future, we will analyze the signal of barcode for many earthquake records, and thus we can correlate the intensity and frequency content of an earthquake to the first set of dis-connectivity.

\section{References}

[1] Loh, C.H., Lee, Z.K., Wu, T.C. and Peng, S.Y. (2000) Ground Motion Characteristics of the Chi-Chi Earthquake of 21 September 1999. Earthquake Engineering and Structural Dynamics, 29, 867-897. http://dx.doi.org/10.1002/(SICI)1096-9845(200006)29:6<867::AID-EQE943>3.0.CO;2-E

[2] Sokolov, V.Y., Loh, C.H. and Wen, K.L. (2002) Comparison of the Taiwan Chi-Chi Earthquake Strong-Motion Data and Ground-Motion Assessment Based on Spectral Model from Smaller Earthquakes in Taiwan. Bulletin of the Seismological Society of America, 92, 1855-1877. http://dx.doi.org/10.1785/0120010178

[3] Sokolov, V.Y., Loh, C.H. and Wen, K.L. (2003) Evaluation of Hard Rock Spectral Models for the Taiwan Region on the Basis of the 1999 Chi-Chi Earthquake Data. Soil Dynamics and Earthquake Engineering, 23, 715-735. http://dx.doi.org/10.1016/S0267-7261(03)00075-7

[4] Chopra, A.K. (1995) Dynamics of Structures: Theory and Applications to Earthquake Engineering. Prentice-Hall, New Jersey.

[5] Kramer, S.L. (1996) Geotechnical Earthquake Engineering. Prentice-Hall, New Jersey.

[6] Kanai, K. (1957) Semi-Empirical Formula for the Seismic Characteristics of the Ground. Bulletin of the Earthquake Research Institute, University of Tokyo, 35, 309-325.

[7] Tajimi, H. (1960) A Statistical Method of Determining the Maximum Responses of a Building Structure during an Earthquake. Proceeding of the 2nd World Conference on Earthquake Engineering, Japan, 781-797.

[8] Liu, C.-S. (2001) Cone of Non-Linear Dynamical System and Group Preserving Schemes. International Journal of Non-Linear Mechanics, 36, 1047-1068. http://dx.doi.org/10.1016/S0020-7462(00)00069-X

[9] Liu, C.-S. and Jhao, W.S. (2014) The Second Lie-Group $S_{o}(n, 1)$ Used to Solve Ordinary Differential Equations. Journal of Mathematic Research, 6, 18-37.

[10] Liu, C.-S. (2014) Disclosing the Complexity of Nonlinear Ship Rolling and Duffing Oscillators by a Signum Function. CMES: Computer Modeling in Engineering \& Sciences, 98, 375-407.

[11] Liu, C.-S. (2015) A Novel Lie-Group Theory and Complexity of Nonlinear Dynamical Systems. Communications in Nonlinear Science and Numerical Simulation, 20, 39-58. http://dx.doi.org/10.1016/j.cnsns.2014.05.004 
Scientific Research Publishing (SCIRP) is one of the largest Open Access journal publishers. It is currently publishing more than 200 open access, online, peer-reviewed journals covering a wide range of academic disciplines. SCIRP serves the worldwide academic communities and contributes to the progress and application of science with its publication.

Other selected journals from SCIRP are listed as below. Submit your manuscript to us via either submit@scirp.org or Online Submission Portal.
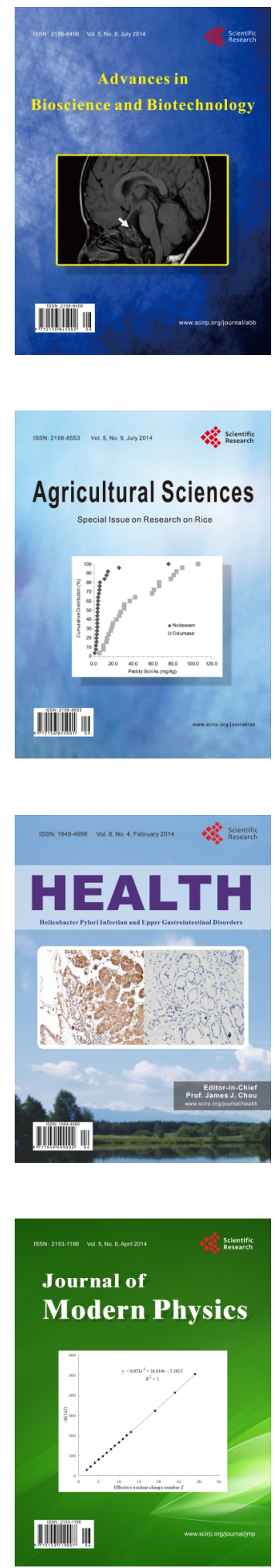
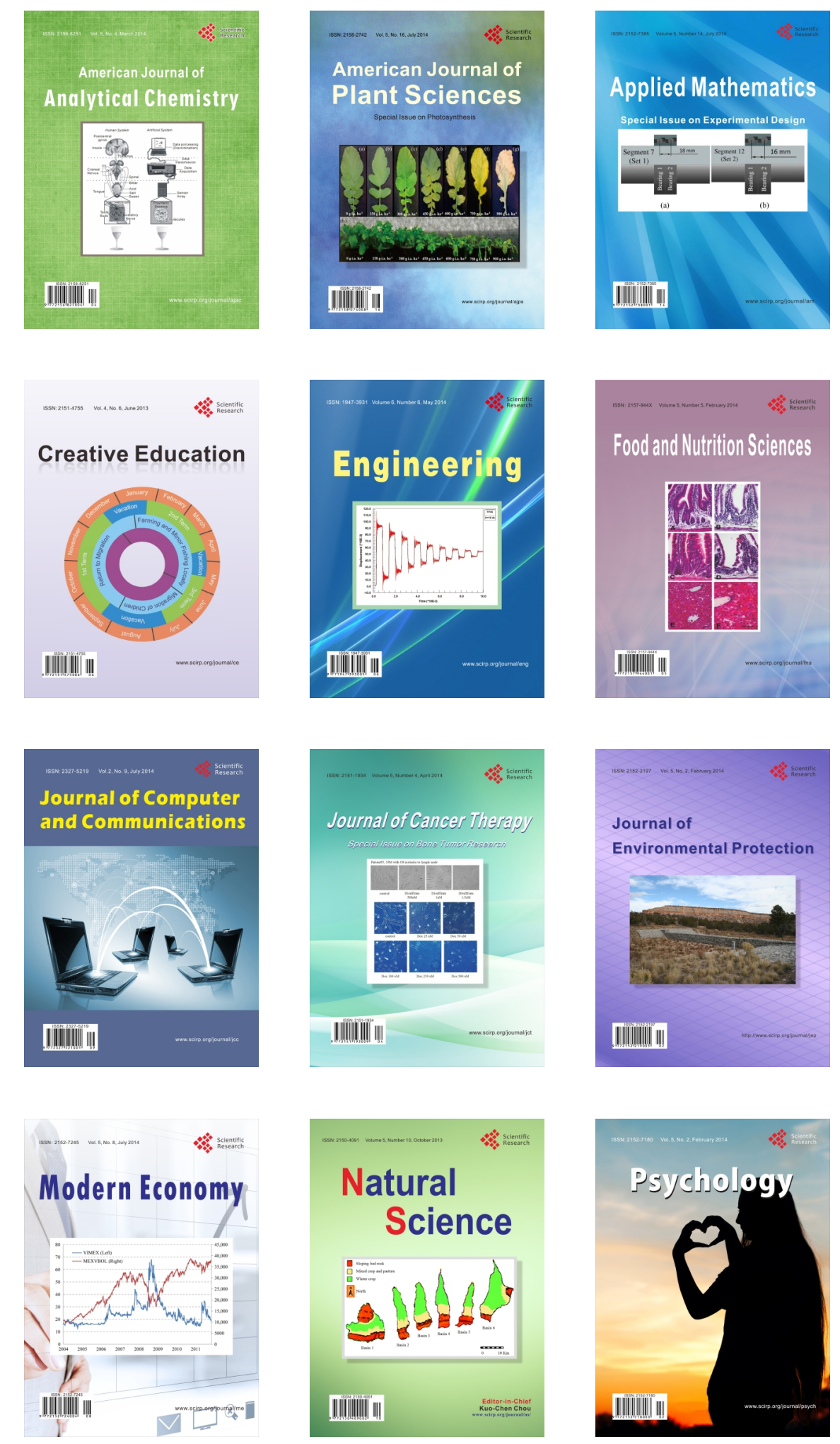